\title{
Akupunktur - wirksam, sicher und natürlich
}

Die Akupunktur ist eine sichere und effektive Therapie. Dies ist eine auch nach den strengen Kriterien der evidenzbasierten Medizin fundierte Aussage.

Es finden sich weltweit 4691 positive Behandlungsempfehlungen in 2308 medizinischen Leit- und Richtlinien (Treatment Guidelines and Clinical Practical Guidelines) für den Einsatz der Akupunktur. Davon betreffen 3117 Empfehlungen den Einsatz der Akupunktur bei insgesamt 149 Indikationen aus dem Schmerzbereich, 1574 Empfehlungen betreffen 157 Nichtschmerzindikationen $[1]^{1}$.

Dies ist eine gewaltige Zahl, und auch wenn der Bezug zur Gesamtanzahl aller weltweit veröffentlichten Leitlinien fehlt, ist diese Tatsache ein überzeugendes Argument für den Einsatz der Akupunktur.

\section{I) Bei nachgewiesenen Indikationen muss jeder Arzt den Patienten über die Option Akupunktur aufklären}

Nach der ärztlichen Berufsordnung besteht eine ärztliche Aufklärungspflicht über Behandlungsalternativen im Rahmen der Sicherungsaufklärung. Deshalb muss der Arzt den Patienten bei Indikationen, bei denen die Akupunktur Evidenzlevel $1 \mathrm{~A}$ erreicht, über die Behandlungsoption mit Akupunktur aufklären. Dies gilt unter anderem für Kopfschmerz, Übelkeit, akuten Schmerz sowie für Schulter-, Rücken- und Knieschmerzen.

Diese Forderung ist nicht nur über die Effektstärke der Akupunktur zu begründen, sondern auch, weil die Akupunktur nachweislich ein sicheres und risikoarmes Verfahren ist. Schwere unerwünschte Wirkungen sind bei sachgemäßer (ärztlicher) Anwendung sehr selten bis extrem selten $(<0,001 \%$; [2]).

\section{Ungerechtferigte Kritik}

Umso seltsamer mutet es an, dass sich eine kleine Gruppe von Menschen auf den Weg gemacht hat, die Akupunktur öffentlich zu diskreditieren. Primär haben sie in den letzten Jahren die Homöopathie angegriffen. Im gleichen Atemzug werden dann aber auch schnell die Naturheilverfahren, weitere Verfahren der Komplementärmedizin und eben auch die TCM inklusive Akupunk-

1 Nach persönlicher Mitteilung von S. Birch wurde nach der hier zitierten Publikation weiterrecherchiert und zum 31.12.2018 gelten die im Text genannten Zahlen.

Deutsche Zeitschrift für Akupunktur 2019 • 62 (1): 1-2

https://doi.org/10.1007/s42212-019-0152-1

๑) Springer Medizin Verlag GmbH, ein Teil von Springer Nature 2019 tur auf eine unsachliche, zynische und teilweise totalitär anmutende Art angegriffen.

Mit undurchsichtigen Mitteln schafft es diese Gruppe in angesehene Zeitungen und Magazine und auch manche Talkshow wurde schon zur Bühne unsachlicher Angriffe. Besondere Aktivität wird im Netz entwickelt, denn kein Artikel über Komplementärmedizin, der nicht sofort kommentiert wird, wenn er nicht den Vorstellungen entspricht.

Diese Attacken werden von einer kleinen Gruppe von Meiningsbildnern unterlegt. Sie fordern lautstark und in völliger Missachtung der wissenschaftlichen Studienlage, der Erfahrung unzähliger Kollegen und der Bedürfnisse der Patienten in der Versorgungsrealität Einschränkungen und Verbote im Bereich der Komplementärmedizin. Hier möchte eine kleine Gruppe den Pluralismus und die im Grundgesetz gesicherte Freiheit der Berufsausübung bzw. die Freiheit der Patienten nach Wahl der Therapie einschränken.

\section{》) Einer sachlichen und wissenschaftli- chen Diskussion stellen sich die Kritiker} nicht

Einer sachlichen und wissenschaftlichen Diskussion stellen sich die Kritiker nicht. Es werden einfach falsche Behauptungen aufgestellt, z. B. „Akupunktur wirkt nicht“, „Homöopathie ist gefährlich“.

Die Strategie der falschen Behauptung, die in der Politik so erfolgreich praktiziert wird, hat nun auch in die Medizin Einzug gehalten. Wir wollen diesem Phänomen nicht zu viel Aufmerksamkeit widmen, denn genau das ist beabsichtigt. Es bleibt nur die Ächtung des Lügners, der sachlich begründete Widerspruch und der schnelle Kommentar im Chatroom.

Arbeiten Sie bitte hier mit, denn in manchen Ländern hat diese totalitäre Bewegung schon echtes Unheil angerichtet. In Spanien droht die Verbannung der Akupunktur aus der Ärztekammer und in Australien der Entzug der Approbation für den akupunktierenden Arzt. Die Bewegung ist international.

Also, beobachten wir aufmerksam und wehren uns gelassen, aber deutlich.

\section{Akupunktur ist natürlich und ökologisch}

Auf einen Punkt sollten wir die Patienten, Kollegen und Entscheider im Gesundheitswesen aufmerksam machen. Die Akupunktur ist eine natürliche Therapie mit hoher ökologischer Verträglichkeit. Wir müssen den Herstellungs- und Entsorgungsprozess diesbezüglich zwar noch genau unter die Lupe nehmen, aber es lässt sich sicherlich schon jetzt behaupten, dass Umweltbelastungen bei Herstellung und Entsorgung von Nadelmaterial 
im Vergleich zu Medikamenten und technischen Therapieverfahren gering sind. Angesichts der bereits jetzt schon vorhandenen Rückstände von Medikamenten im Trinkwasser und aufwendiger, ressourcenverbrauchendenr Herstellung mancher medizinischer Verfahren ist die anznehmende gute Umweltverträglichkeit der Akupunktur ein relevanter Vorteil.

\section{9 - Was steht an?}

Es wird sicherlich wieder ein spannendes Akupunkturjahr. Wir erwarten neue Studienergebnisse aus vielen Nicht-Schmerzindikationen, denn einige große Studien stehen kurz vor Abschluss, z. B. im Bereich der Psychiatrie und Immunologie.

Die Implementierung der Akupunktur in die Klinikroutine schreitet voran.

Das aktuelle Beispiel ist die Integration der Akupunktur in die chirurgische und anästhesiologische perioperative Versorgung in den Ambulatorien und Kliniken.

Für die Lehrenden steht in diesem Jahr die Überarbeitung des (Muster-)Kursbuches an. Es wird einige wenige Anpassungen geben, die neuen Erkenntnissen geschuldet sind. Hier werden, wie immer in den letzten Jahren, die Akupunkturgesellschaften gemeinsam und partnerschaftlich daran arbeiten.

Eines ist sicher: Wir werden auch in diesem Jahr die chinesische Strategie der Belt-and-Road-Politik zu spüren bekommen.

Ziel ist es unter anderem, die chinesische Akupunktur in der ganzen Welt zu verbreiten. Im Handel mit Deutschland heißt dies Austausch von TCM gegen den Import von Industriegütern, z. B. Autos.

Es sollen flächendeckend Kooperationen und Kliniken installiert werden. Es werden Masterstudiengänge angeboten, deren Inhalt die chinesische Seite vorgibt. Es sollen noch mehr nichtärztliche chinesische Therapeuten installiert werden. All diese Vereinbarungen laufen über das Wirtschaftsministerium, nicht über das Gesundheitsministerium. So manche Universität ist schon auf das zunächst verlockend ausschauende Angebot der Lehrkooperation mit chinesischen Universitäten hereingefallen. Wir beobachten diese Entwicklung selbstbewusst im Wissen um die wesentlichen Erkenntnisse der westlichen Akupunkturweiterentwicklung.

\section{I) Wir kooperieren gerne bei interessan- ten Forschungs- und Lehrprojekten}

Wir kooperieren gerne bei interessanten Forschungs- und Lehrprojekten auf Augenhöhe. Die Besuche von TCM-Zentren und Akupunkturkliniken in China unterstützen wir sehr. Das Mutterland der Akupunktur hat viel zu bieten. Wir haben großen Respekt vor den Errungenschaften der TCM und sind lernbereit.

Einer einseitig definierten, kommerzialisierten und fast schon kolonialistisch anmutenden Verbreitung der chinesischen Medizin, die die ärztliche Kompetenz und westliche medizinische Errungenschaften in Diagnostik und Therapie negiert, erteilen wir eine klare Absage.

Setzen wir unsere Energie vielmehr dafür ein, eine moderne Akupunktur in die Hörsäle zu bringen und dafür zu sorgen, dass jeder Arzt die Möglichkeiten und die Grenzen der Akupunktur einschätzen kann. Setzen wir uns für die Qualität in der Akupunkturanwendung ein und für eine angemessene Honorierung.

Zeigen wir die Möglichkeiten einer ganzheitlichen Therapie auf, die physiologische Wirkungen, eine optimierte PatientenArzt-Beziehung und ein psychosomatisches Grundverständnis auf das Beste zum Wohle des Patienten vereint.

Ein schönes Ziel für 2019!

Ihr

Dominik Irnich

1. Vorsitzender DÄGfA

Literatur

1. Birch S, Lee MS, Alraek T, Kim TH (2018) Overview of treatment guidelines and clinical practical guidelines that recommend the use of acupuncture: A Bibliometric analysis. J Altern Complement Med 24(8):752-769. https://doi.org/10.1089/ acm.2018.0092

2. Witt CM, Pach D, Brinkhaus B, Wruck K, Tag B, Mank S, Willich SN (2009) Safety of acupuncture: Results of a prospective observational study with 229,230 patients and introduction of a medical information and consent form. Forsch Komplementmed 16(2):91-97

\section{Korrespondenzadresse}

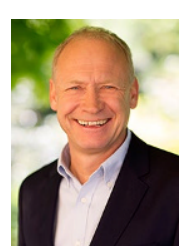

PD Dr. Dominik Irnich

Interdisziplinäre Schmerzambulanz, Klinikum der Ludwig-Maximilians-Universität München (LMU), Campus Innenstadt

Pettenkoferstr. 8a, 80366 München, Deutschland

Dominik.Irnich@med.uni-muenchen.de

Interessenkonflikt. D. Irnich erhält auschließlich Aufwandsentschädigungen von öffentlichen Institutionen, Vereinen und Verlagen für Vorträge, Lehrtätigkeit und Publikationen im Bereich der Schmerztherapie und Komplementärmedizin inklusive Akupunktur und TCM und für ehrenamtliche Tätigkeiten. Es besteht keine honorierte Berater-, Vortrags- oder Forschungstätigkeit im Zusammenhang mit Hersteller- oder Industrieinteressen. 